\title{
IS element IS16 as a molecular screening tool to identify hospital-associated strains of Enterococcus faecium
}

\author{
Guido Werner $^{1 *}$, Carola Fleige ${ }^{1}$, Uta Geringer ${ }^{1}$, Willem van Schaik², Ingo Klare ${ }^{1}$ and Wolfgang Witte
}

\begin{abstract}
Background: Hospital strains of Enterococcus faecium could be characterized and typed by various molecular methods (MLST, AFLP, MLVA) and allocated to a distinct clonal complex known as MLST CC17. However, these techniques are laborious, time-consuming and cost-intensive. Our aim was to identify hospital E. faecium strains and differentiate them from colonizing and animal variants by a simple, inexpensive and reliable PCR-based screening assay. We describe here performance and predictive value of a single PCR detecting the insertion element, IS16, to identify hospital E. faecium isolates within a collection of 260 strains of hospital, animal and human commensal origins.
\end{abstract}

Methods: Specific primers were selected amplifying a 547-bp fragment of IS16. Presence of IS16 was determined by PCR screenings among the 260 E. faecium isolates. Distribution of IS16 was compared with a prevalence of commonly used markers for hospital strains, esp and hyl Efm. All isolates were typed by MLST and partly by PFGE. Location of IS16 was analysed by Southern hybridization of plasmid and chromosomal DNA.

Results: IS16 was exclusively distributed only among 155 invasive strains belonging to the clonal complex of hospital-associated strains ("CC17"; 28 MLST types) and various vancomycin resistance genotypes (vanA/B/negative). The five invasive IS16-negative strains did not belong to the clonal complex of hospital-associated strains (CC17). IS16 was absent in all but three isolates from 100 livestock, food-associated and human commensal strains ("nonCC17"; 64 MLST types). The three IS16-positive human commensal isolates revealed MLST types belonging to the clonal complex of hospital-associated strains (CC17). The values predicting a hospital-associated strain ("CC17") deduced from presence and absence of IS16 was 100\% and thus superior to screening for the presence of esp (66\%) and/or hyl Efm (46\%). Southern hybridizations revealed chromosomal as well as plasmid localization of IS16.

Conclusions: This simple screening assay for insertion element IS16 is capable of differentiating hospital-associated from human commensal, livestock- and food-associated E. faecium strains and thus allows predicting the epidemic strengths or supposed pathogenic potential of a given E. faecium isolate identified within the nosocomial setting.

\section{Background}

Vancomycin- and multi-resistant Enterococcus strains, especially strains of $E$. faecium, raise major concerns in intensive care medicine due to limited treatment options [1]. E. faecium is ecologically widely distributed and expected to play a central role as a reservoir and "turntable" for antibiotic resistance determinants in the bacterial world, and especially among Gram-positive

\footnotetext{
* Correspondence: wernerg@rki.de

${ }^{1}$ Robert Koch Institute, Wernigerode Branch, Wernigerode, Germany

Full list of author information is available at the end of the article
}

bacteria [2]. A number of molecular typing and characterization techniques such as AFLP, MLVA and MLST and comparative genomic hybridizations allowed differentiating strains of E. faecium and allocating them into various clonal complexes as based on core as well as accessory genome content [3,4]. Isolates belonging to hospital-associated clonal types (for instance, MLST CC17) could be identified and a supposed enhanced spreading potential among the nosocomial setting for isolates of this specific subgroup is predictable [4]. Reports from recent years described increasing annual rates of E. faecium bacteraemia in European countries

\section{Biomed Central}


$[5,6]$. When investigated in greater detail, the increase was due to increasing numbers of hospital-associated clonal types of E. faecium (MLST CC17) whereas numbers of other clonal types remained constant over time again emphasizing the aforementioned increased potential for nosocomial spread [5]. The molecular techniques to identify hospital-associated E. faecium strains are laborious, time-consuming and cost-intensive and are thus not applicable for routine diagnostics. Results of recent bacterial, epidemiological, microarray-based and genomic studies suggest that a number of markers are enriched among hospital strains of $E$. faecium including collagen adhesion factors $\mathrm{acm}$ and $\mathrm{scm}[7,8]$, other matrix-binding proteins and pili $[9,10]$, a supposed hyaluronidase, $h y l_{E f m}[11,12]$, biofilm-associated markers including the enterococcal surface protein gene, esp $[13,14]$, and various genomic islands encoding proteins of unknown functions $[15,16]$. Having acquired a number of these aforementioned determinants, these strains may have an increased pathogenic potential $[4,10,17]$. However, distribution of these markers among hospital strains is not exclusive and thus the predictive value is limited in terms of specificity and sensitivity. Certain markers possess pseudogenes and partly deleted variants complicating establishing a simple PCR based screening test $[8,17]$. Acquired ampicillin resistance appeared as a phenotypic trait of hospital strains, at least in Europe; however, this feature is also prevalent among non-hospital strains in some parts of the world, such as Asia and North America [18-20]. Microarray based genomic comparisons revealed a specific mobile element, insertion element IS16, exclusively prevalent among hospital E. faecium strains from an international collection of isolates [3]. In the present study, we established a PCRbased IS16 screening test and evaluated its robustness, sensitivity, specificity and predictive value for 260 precharacterized isolates of E. faecium from different human and animal sources. IS16 was localized in a subset of E. faecium isolates $(\mathrm{n}=45)$ representing 29 MLST types by Southern hybridization. Available E. faecium genome data were examined for the presence of IS16 homologous sequences and a genetic association of IS16 with genomic markers is discussed.

\section{Methods}

\section{Strain collection}

Our laboratory serves as a Focal Laboratory for Enterococci in Germany. In general, enterococcal isolates sent to us are pre-selected for being vancomycin- and multiresistant or with the suspicion of clonal spread (local, regional, and country-wide outbreak analysis). Our collection of E. faecium test strains included 160 invasive isolates (blood cultures) from 60 different German diagnostic or hospital laboratories isolated between 1995 and 2007. Blood culture strains were pre-selected by geography and hospital origin, year of isolation, and different van genotypes $(v a n-/ A / B)$. The blood culture isolates were compared against 68 human commensal (outpatient) isolates from four different prevalence studies performed in different parts of Germany (1996, $1997,1999,2003)[24,25]$ and 32 isolates from animal (chicken, pigs) and meat samples (broiler, pork) (1994, 1995, 1999) [21-23]. In all cases duplicate isolates were excluded based on clinical/epidemiological data (same patient/hospital or animal sample/flock) and of molecular typing results (for instance, based on SmaImacrorestriction using PFGE for human commensal isolates [24,25]; unpublished results). Antibiotic susceptibilities were determined by microbouillon dilution in $\mathrm{MH}$ bouillon. Ampicillin resistance is defined by an MIC of $>8 \mathrm{mg} / \mathrm{L}$ and vancomycin resistance by $>4 \mathrm{mg} / \mathrm{L}$ (http:// www.eucast.org). Summarized data are presented in Table 1 and detailed information is given in Additional files 1, 2, 3. Ethical approval was not needed for this study; all strains were pre-selected and were received anonymously from German microbiological or hospital laboratories.

\section{DNA isolation}

Genomic DNA was prepared using a DNA extraction kit (DNeasy Tissue Kit; Qiagen, Hilden, Germany) according to the manufacturer's instructions. An initial cell wall lysis step was added dissolving the cell pellet in TES buffer [10 mM Tris, $0.5 \mathrm{mM}$ ethylene diamine tetra-acetic acid (EDTA), 10\% sucrose ( $\mathrm{pH}$ 8.0)] plus $10 \mathrm{mg} / \mathrm{mL}$ lysozyme (Roche Applied Science, Mannheim, Germany) followed by incubation at $37^{\circ} \mathrm{C}$ for 30 min. Plasmid DNA was isolated using a common phenol/chloroform-based alkaline lysis strategy [26].

\section{PCR}

PCR was performed with illustra ${ }^{\mathrm{TM}}$ PuRe Taq Ready-ToGo $^{\text {TM }}$ PCR Beads (GE Healthcare, Freiburg, Germany) according to the manufacturer's instructions. Approximately $0.5 \mu \mathrm{L}$ of isolated genomic DNA (ca. $10 \mathrm{ng}$ ) and primers (200 nM each) were added. Amplification of fragments representing the esp, $h y l_{E f m}$ and $v a n A / B$ genes was performed in a multiplex PCR as described elsewhere [27]. The following strains and plasmids were used as positive control samples: plasmid pRUM (IS16), plasmid pLG1 $\left(h y l_{E f m}\right)$; plasmid pIP816 (vanA; E. faecium BM4147), E. faecium U0317 (esp), and E. faecalis V583 (vanB). E. faecalis OG1RF served as a negative control sample for all PCR assays. PCR primers and conditions for screening IS16 elements were as follows: IS16-F: 5'-CATGTTCCACGAACCAGAG and IS16-R: 5'-TCAAAAAGTGGGCTTGGC, annealing temperature was $53^{\circ} \mathrm{C}$, the predicted size of the product was $547 \mathrm{bp}$ 
Table 1 Prevalence of epidemic markers among E. faecium isolates from different origins (see also Figure 1 and Additional Files 1, 2, 3)

\begin{tabular}{|c|c|c|c|c|c|c|c|}
\hline Origin & $\begin{array}{l}\text { No. of } \\
\text { isolates }\end{array}$ & $\begin{array}{l}\text { No. of MLST } \\
\text { types }\end{array}$ & AmpR & $\begin{array}{c}\text { PCR IS16 } \\
+\end{array}$ & $\begin{array}{c}\text { PCR esp } \\
+\end{array}$ & $\begin{array}{c}\text { PCR hyl } \\
+ \\
+\end{array}$ & $\begin{array}{c}\text { PCR van } A+/ B \\
+\end{array}$ \\
\hline Blood cultures & 160 & 32 & $\begin{array}{c}98.1 \% \\
(n=157)\end{array}$ & $\begin{array}{c}96.9 \% \\
(n=155)\end{array}$ & $\begin{array}{c}63.8 \% \\
(n=102)\end{array}$ & $\begin{array}{c}45.6 \% \\
(n=73)\end{array}$ & $\begin{array}{c}58.1 \% \\
(n=93)\end{array}$ \\
\hline 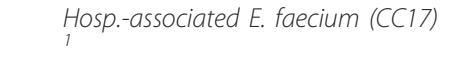 & 155 & 28 & $\begin{array}{c}100 \% \\
(n=156)\end{array}$ & $\begin{array}{c}100 \% \\
(n=156)\end{array}$ & $\begin{array}{c}65.8 \% \\
(n=102)\end{array}$ & $\begin{array}{l}46.5 \% \\
(n=72)\end{array}$ & $\begin{array}{l}58.7 \% \\
(n=91)\end{array}$ \\
\hline Human commensal & 68 & 46 & $\begin{array}{c}2.9 \% \\
(n=2)\end{array}$ & $\begin{array}{c}4.4 \% \\
(n=3)^{2}\end{array}$ & $0 \%$ & $0 \%$ & $\begin{array}{c}14.7 \% \\
(n=10)\end{array}$ \\
\hline Animal commensal and meat sources & 32 & 22 & $\begin{array}{c}6.2 \% \\
(n=2)\end{array}$ & $0 \%$ & $0 \%$ & $0 \%$ & $\begin{array}{c}40.6 \% \\
(n=13)\end{array}$ \\
\hline
\end{tabular}

BC MLST CC17 (second line) shown in italics $(n=155)$ is a subgroup of all blood culture $E$. faecium isolates presented in the first line representing all isolates allocated to the clonal complex of hospital-aascoiated strains (CC17).

AmpR, ampicillin resistance; ${ }^{1}$ including two isolates of ST65 representing a singleton but known to be found only among clinical strains; ${ }^{2}$ all three belonged to the clonal complex of hospital-associated strain types (MLST CC17; 2x ST18, 1x ST413; see also Additional file 4).

(reference E. faecium plasmid pRUM; GenBank acc. no. AF507977). Primers were established using software Acelrys DS Gene 1.5.

\section{PFGE typing}

Genomic DNA for PFGE analysis was isolated and treated as described recently [27]. The agarose gel concentration was $1 \%$, the CHEF-DR III apparatus (Bio-Rad Laboratories, Hercules, CA, USA) was used for PFGE. The ramped pulsed times were as follows: 1 - $11 \mathrm{sec}$ for $15 \mathrm{~h}$ and $11-30 \mathrm{sec}$ for $14 \mathrm{~h}$ at $14^{\circ} \mathrm{C}$. SmaI-digested Staphylococcus aureus NCTC 8325 was used as a molecular mass standard on all PFGE gels. Chromosomal DNA of E. faecium strains was digested with I-Ceu-I for $16 \mathrm{~h}$ at $37^{\circ} \mathrm{C}$. Plasmids were linearized by $\mathrm{S} 1$ nuclease (TaKaRa, Germany) treatment for $15 \mathrm{~min}\left(37^{\circ} \mathrm{C}\right)$ and subsequent resolution of the linearized plasmid DNA in PFGE. The ramped pulsed times for I-Ceu-I gels were $5-30 \mathrm{sec}$ for $22 \mathrm{~h}$ and for S1 PFGE 5 - $35 \mathrm{sec}$ for $22 \mathrm{~h}$ at $14^{\circ} \mathrm{C}[28]$.

\section{Southern hybridizations}

Southern hybridization experiments were done as described elsewhere using a PCR-generated digoxigeninlabelled IS16 probe (DIG High Prime; Roche Applied Science, D), hybridization chemicals and equipment from commercial kits and according to recommendations of the manufacturer (Roche, Germany). Immunological detection was done as recommended using a chemiluminescent probe (CDP-Star ${ }^{\mathrm{TM}}$, Roche, D) and several readouts were taken at 10, 30, 60 and $120 \mathrm{~min}$ in a chemi-imager from Bio-Rad (Chemidoc XRS, BioRad Labs., Hercules, US).

\section{MLST and DNA sequencing}

PCRs amplifying the seven loci used for MLST were done according to the reference (http://efaecium.mlst. net/). Sequencing reactions were performed according to the manufacturer's recommendations for cycle sequencing of PCR products (Applied Biosystems, Germany). Sequence files were read, evaluated, aligned and compared to the reference set of alleles using sequencing software Lasergene 8.0 from DNA-STAR (SeqMan 8.0; EditSeq 8.0), TraceEditPro v. 1.1.1 from Ridom (http://www.ridom.de), and via the official MLST webpage (http://efaecium.mlst.net/). Phylogenetic trees were generated using software programmes eBURST [29] for the set of determined MLST types and global optimised eBURST [30] for the entire set of all available 524 MLST types (http://efaecium.mlst.net/).

\section{Statistics}

Statistical analyses were performed with software package EpiCompare 1.0 (Ridom).

\section{Results}

Identification of IS16 primer sequences

Based on available sequences (pRUM [31] or composite $(v a n B)$ transposons [32]) we established PCR primers for an IS16-specific internal fragment (547 bp). Specificity and sensitivity was evaluated against available bacterial databases (BlastN, GenBank). The PCR was established using DNA from reference samples known to be positive for IS16 (plasmid pRUM) and from negative control samples known to lack IS16 (E. faecalis OG1RF). PCR results were as expected and amplified products from reference samples were sequenced for confirming specificity of the amplified PCR product (not shown).

\section{Screening for markers in the two sets of strains}

Genomic DNA was screened by PCR using the established PCR primers for IS16, esp and $h y l_{E f m}$. IS16 was highly prevalent among invasive E. faecium (n = 155/ 160; Table 1$)$ and mainly absent $(n=3 / 100)$ from human commensal or animal and meat sources. 
The three IS16-positive commensal isolates were from humans. Other markers supposedly enriched among clinical or hospital-associated E. faecium isolates were also assessed: the esp gene was prevalent among $64 \%$ of all blood culture isolates and $46 \%$ of all invasive isolates were $h y l_{E f m}$-positive (Table 1). None of the 100 nonclinical strains possessed esp or $h y l_{E f m}$. Ampicillin resistance was prevalent among almost all clinical strains (98\%). Two human commensal and two animal isolates were also ampicillin-resistant.

\section{MLST typing}

All 260 E. faecium isolates were MLST typed. Discriminatory power among clinical isolates $(\mathrm{n}=160$ isolates; 32 MLST types DI $=0.88995 \%$ CI [0.864 - 0.913]) was much less than among human and animal commensal isolates $(\mathrm{n}=100$ isolates, 64 MLST types; DI $=0.988$; 95\% CI [0.983 - 0.994]). This suggests that prevalent hospital-associated clonal types differ less than their colonizing counterparts in animals and humans. In fact, 126 of the 160 invasive E. faecium isolates belonged to only eight MLST types known to represent major hospital-associated clonal types (ST16, ST17, ST18, ST78, ST117, ST192, ST202, ST203) whereas the other 24 MLST types occurred only occasionally. Based on their MLST types and subsequent eBURST and goeBURST analyses, isolates could be sorted into different clonal complexes which were in agreement with their corresponding ecological background (compare also to [4]). Figure 1 shows eBURST trees generated with all MLST types determined in this study. A phylogenetic tree of all currently detached MLST types ( $\mathrm{n}=524$; 21.04.2010; http://efaecium.mlst.net/) using goeBURST is shown as Additional File 4. Based on these two calculations, almost all $(\mathrm{n}=155 / 160)$ invasive isolates belonged to the clonal complex of hospital-associated strains (CC17 + ST65 [singleton]). The five IS16-negative clinical isolates represent colonizing strain types not associated with clonal types of hospital strains ("nonCC17"; ST9 [n = 2], ST104; ST271; ST374). The majority of commensal isolates represented many individual MLST types but belonging to the major clonal complexes of animal and human colonizing strain types

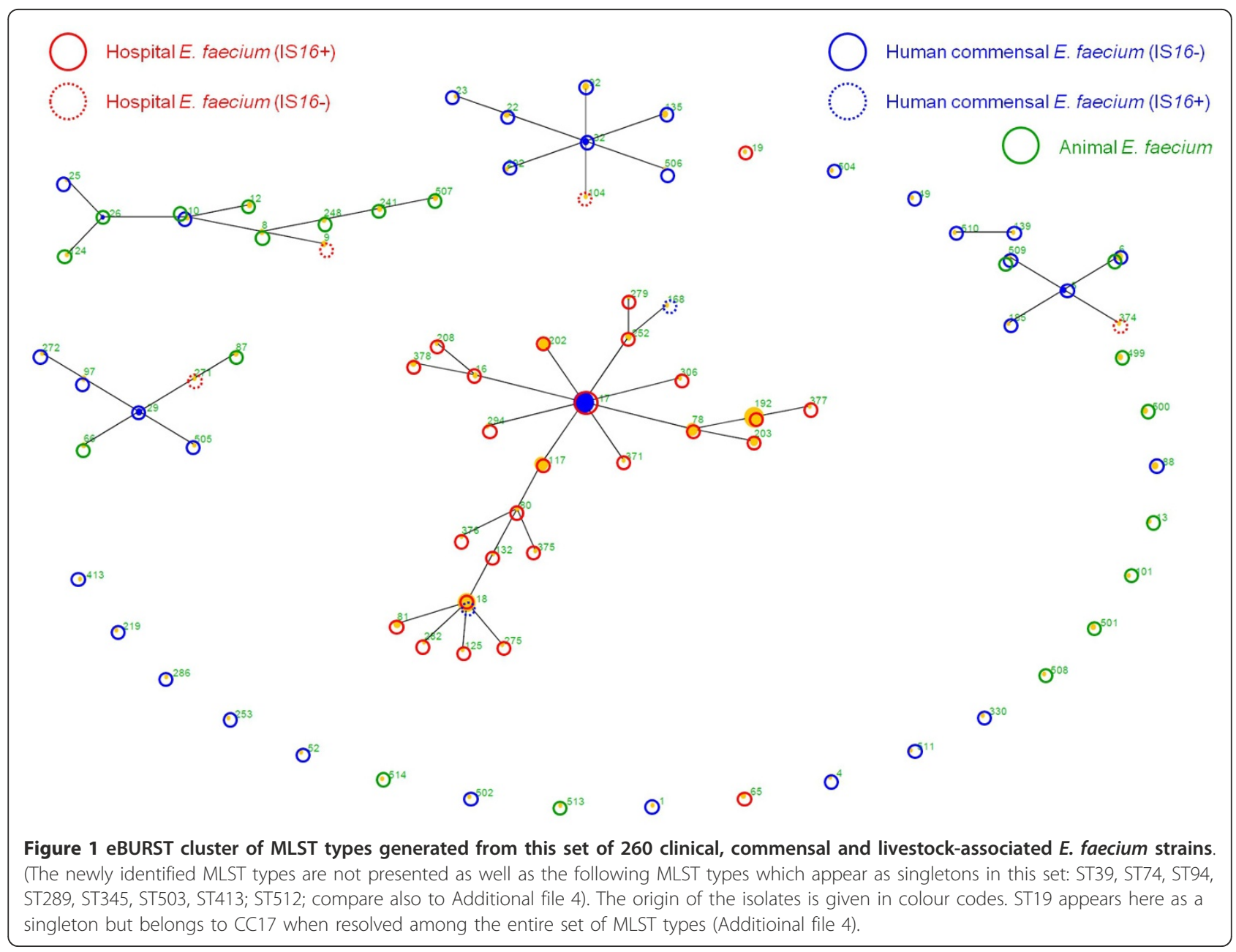


(CC5, CC22/36, CC1). The three IS16-positive commensal E. faecium isolates revealed MLST types (ST18, ST413) belonging to the clonal complex of hospitalassociated strains (CC17).

\section{Southern hybridizations of IS16 probes}

The localization of IS16 among 45 representative E. faecium isolates from various origins and altogether 29 different MLST types was investigated. I-Ceu-I digested chromosomal DNA resolved in PFGE and plasmid DNA analysed inS1 PFGE and common agarose gels were transferred onto nylon membranes and hybridized against a labelled IS16 probe. Results are shown in Table 2. If available, several representatives of identical MLST types differing in their van gene content were considered for analysis (see for instance ST17 van $A / B /$ negative isolates). In general, the IS16 probe hybridized to (up to three) chromosomal bands suggesting several copies of the same element present in the genome (a single hybridizing chromosomal band could still result from several IS16 copies on it). Four isolates (ST17, ST81, ST202, ST275) showed IS16 only hybridizing to plasmid bands. Twenty-two strains $(n=22 / 45)$ revealed a signal for both chromosomal as well as plasmid bands. Hybridization patterns were independent of the van gene content (Table 2).

\section{Screening $E$. faecium genomes for IS16}

Several E. faecium genomes are available as genomic fragments for preliminary analysis (collection I: seven $E$. faecium genomes; [33]; collection II: eleven E. faecium isolates from the Human Microbiome Project; (http:// nihroadmap.nih.gov/hmp/; http://www.ncbi.nlm.nih. gov//genomes/geblast.cgi?gi=6511\#SearchSet). A BLAST search for IS16-related sequences in both E. faecium collections revealed several hits. However, as far as some detailed information was available, the neighbouring DNA fragments all showed different genetic links. IS16 was prevalent among four out of seven genomes in collection I [33]. IS16 was linked to (a) several putative fragments of a conjugative transposon (strain E1162, ST17), (b) ORF involved in sugar metabolism (strain E1636; ST106), (c) a genomic fragment containing other transposases of the IS256 family (strain U0317, ST78) and (d) the supposed pathogenicity island containing the esp gene (strain E1679, ST104). None of the other two strains also containing the esp PAI possessed IS16 within its structure. In the deposited genomic fragments from the Human Microbiome Project, IS16 was infrequently identified, for instance, in E. faecium 1,230,933 linked to a metal-dependent hydrolase or in E. faecium TC6 linked to an extracellular solute-binding protein, family 1 . In E. faecium DO a 5' link to a C4-dicarboxylate anaerobic carrier gene and a 3' link to a
Table 2 Localization of IS16 to I-Ceu-I digested chromosomal DNA resolved in PFGE and plasmid DNA as based on Southern hybridizations

\begin{tabular}{|c|c|c|c|c|c|}
\hline $\begin{array}{l}\text { Strain } \\
\text { no. }\end{array}$ & Origin & $\begin{array}{l}\text { MLST } \\
\text { type }\end{array}$ & $\begin{array}{c}\text { van } \\
\text { genotype }\end{array}$ & $\begin{array}{l}\text { I-Ceul } \\
\text { PFGE }\end{array}$ & Plasmid \\
\hline UW 6714 & $B C$ & 202 & - & $-*$ & + \\
\hline UW 1245 & $B C$ & 202 & $\operatorname{van} A$ & - & + \\
\hline UW 6293 & $B C$ & 202 & $\operatorname{van} B$ & $-^{*}$ & $-\#$ \\
\hline UW 6295 & $B C$ & 202 & $\operatorname{van} B$ & $-^{*}$ & $-\#$ \\
\hline UW 4962 & $B C$ & 203 & $\operatorname{van} A$ & + & $-\#$ \\
\hline UW 6989 & $B C$ & 203 & - & + & ${ }^{\#}$ \\
\hline UW 6502 & $B C$ & 208 & $\operatorname{van} B$ & + & + \\
\hline UW 5918 & $B C$ & 252 & vanA & + & + \\
\hline UW 4675 & $B C$ & 252 & $\operatorname{van} B$ & + & - \\
\hline UW 6617 & $B C$ & 275 & $\operatorname{van} A$ & - & + \\
\hline UW 4960 & $B C$ & 279 & $\operatorname{van} B$ & + & - \\
\hline UW 6033 & $B C$ & 282 & vanA & + & + \\
\hline UW 4512 & $B C$ & 294 & - & + & + \\
\hline UW 6297 & $B C$ & 306 & - & + & + \\
\hline UW 6935 & $\mathrm{BC}$ & 371 & - & + & + \\
\hline UW 3142 & $B C$ & 375 & $\operatorname{van} A$ & + & + \\
\hline UW 5275 & $B C$ & 376 & $\operatorname{van} B$ & + & + \\
\hline UW 6983 & $B C$ & 377 & - & + & + \\
\hline UW 6112 & $B C$ & 378 & - & + & + \\
\hline UW 4208 & $B C$ & New & - & + & + \\
\hline UW 6642 & $B C$ & New & vanA & + & + \\
\hline AK-EM 40 & $\mathrm{HC}$ & 18 & - & + & + \\
\hline AK-EM 53 & $\mathrm{HC}$ & 413 & - & + & + \\
\hline K226.7 & $\mathrm{HC}$ & 18 & - & + & - \\
\hline UW 2055 & $B C$ & 16 & $\operatorname{van} A$ & $-*$ & + \\
\hline UW 3695 & $B C$ & 17 & - & + & + \\
\hline UW 3544 & $B C$ & 17 & $\operatorname{van} A$ & + & $-\#$ \\
\hline UW 5352 & $B C$ & 17 & $\operatorname{van} B$ & - & + \\
\hline UW 6990 & $B C$ & 18 & & + & + \\
\hline UW 1952 & $B C$ & 18 & vanA & + & + \\
\hline UW 2900 & $\mathrm{BC}$ & 18 & $\operatorname{van} B$ & + & $-\#$ \\
\hline UW 1218 & $B C$ & 19 & $\operatorname{van} A$ & + & ${ }^{\#}$ \\
\hline UW 3056 & $B C$ & 65 & - & + & + \\
\hline UW 6880 & $B C$ & 78 & - & + & + \\
\hline UW 4671 & $B C$ & 78 & vanA & + & - \\
\hline UW 2457 & $B C$ & 80 & vanA & + & - \\
\hline UW 6985 & $B C$ & 81 & - & - & + \\
\hline UW 2771 & $B C$ & 81 & $\operatorname{van} A$ & + & - \\
\hline UW 6882 & $B C$ & 117 & - & + & - \\
\hline UW 1983 & $B C$ & 117 & $\operatorname{van} A$ & + & - \\
\hline UW 6805 & $B C$ & 125 & $\operatorname{van} A$ & + & + \\
\hline UW 2824 & $B C$ & 132 & $\operatorname{van} A$ & + & -\# \\
\hline UW 1716 & $B C$ & 192 & - & + & $-^{\#}$ \\
\hline UW 5852 & $B C$ & 192 & vanA & + & + \\
\hline UW 5267 & $B C$ & 192 & $\operatorname{van} B$ & + & $-\#$ \\
\hline
\end{tabular}

If available, different representatives of identical MLST types were considered (see for instance ST17 vanA/B/-negative isolates).

PFGE, pulsed-field gel electrophoresis; MLST, Multi-locus sequence typing; BC, blood culture; HC, human commensal; *, Hybridization signal with PFGE slot; \#, negative results in repeated experiments. 
chloramphenicol actetyltransferase resistance gene followed by a replicase and relaxase genes indicated a supposed plasmid origin of this contig (not shown in details). Available sequence data allow extracting the MLST type related allele information out of the sequenced genomes and a corresponding analysis was done on the seven (collection I) and eleven (collection II) accessible genomes. Of the 11 isolates positive for IS16 eight strains possessed hospital-associated MLST types (ST16, ST17, ST18, ST78, ST203, ST-NEW) and three did not belong to clonal complexes of hospitalassociated types (ST25, ST104, ST114). Intriguingly, of the latter three, one was a clinical isolate also positive for esp, a marker commonly associated only with hospital-associated strains.

\section{Epidemiological analysis of clinical strains}

We introduced PCR-based screening for IS16 for all E. faecium isolates sent to our Focal Laboratory for Enterocooci in Germany in late 2007. In 2008 we received $314 \mathrm{E}$. faecium isolates (from $>57$ hospitals located in 13 of the 16 German federal states; all VRE) and in 2009334 E. faecium isolates (from $>49$ hospitals located in 11 German federal states, 90\% VRE) from clinical sources for further analyses. Altogether $99.4 \%$ $(\mathrm{n}=312 / 314)$ in 2008 and $97.3 \%(\mathrm{n}=325 / 334)$ in 2009 were IS16-positive demonstrating wide prevalence of multi-resistant (VRE), hospital-associated strain types in hospitals in Germany.

\section{Discussion}

Early recognition of epidemic E. faecium strains is critical for a timely introduction of strategies to prevent and control their further spread. Hospital-associated clonal types of E. faecium often disseminate unrecognized among the nosocomial setting before and until first VRE cases suddenly appear $[5,6]$. But standardised methods for rapid diagnostics of these hospital-associated E. faecium strains are missing and modern, DNA sequence-based typing techniques are laborious, time-consuming and comparably expensive. Acquired ampicillin resistance $[5,6,34]$ and high-level ciprofloxacin resistance $[35,36]$ appear as phenotypic traits of hospital-associated strains. However, ampicillin resistance in animal or commensal enterococci is also prevalent to a certain extent [37], at least in many North American and Asian countries [38] and the predictive value of acquired ciprofloxacin resistance is limited since it accumulates over time and differently in various clonal types of hospital strains [36]. Several genetic markers, such as esp, $h y l_{E f m}$ or the purK1 allele (used as part of the MLST scheme) are not ubiquitous traits of all hospital-associated E. faecium strains and failure to detect them does not reliably indicate a strain with a limited spreading or pathogenic potential [27,34,36,39-41]. It was also shown recently that $h y l_{E f m}$ is plasmid-located $[11,42]$, transferable in vitro into several non-hospital $E$. faecium strains and thus cannot be suggested to serve as a specific marker to predict a clonal background of a given strain [43]. A reliable and rapid molecular test to differentiate commensal from hospital-acquired strains is desirable; results from comparative genomic hybridizations and genome sequencing projects show promising candidate markers including the described insertion element IS16 [3].

According to our data, IS16 is highly specific for predicting hospital-associated strain types (Table 1; Additional files 1, 2, 3). Altogether 155 of the 160 blood culture isolates from 1995 to 2007 from $>50$ contributing laboratories or hospitals and representing 28 MLST types were all IS16 positive. The comparably high number of (a) participating hospitals/laboratories contributing strains, (b) blood culture strains representing many different MLST types and different van genotypes, and (c) isolated from over 13 years compensates for a geographical limitation of a strain collection that only included isolates from Germany. The presence of IS16 was independent of resistance gene clusters known to possess this element; only 19 isolates were $v a n B$-positive (12\%) and the remaining isolates were vancomycin susceptible $(\mathrm{n}=67 ; 42 \%)$ or of the vanA-type $(\mathrm{n}=74 ; 46 \%)$. Since it is known from previous plasmid analysis that IS16 could be associated with specific plasmid types (e.g., pRUM), we identified the location of IS16 in a representative set of E. faecium isolates (vanA/ $B /$-negative; 29 MLST types; Table 2). Southern hybridizations revealed chromosomal, plasmid and both localizations for IS16 suggesting a somehow restricted mobile gene pool within hospital-associated clonal types. All but three investigated human commensal and all animal strains possessed MLST types different from hospital-associated strains; all but the three aforementioned strains were IS16 negative. The three human commensal isolates possessing IS16 revealed MLST types ST18 and ST413, allocating them to hospital-associated strains and again emphasising the excellent predictive value of this element for identifiying hospital-associated strain types. PCRs for esp and $h y l_{E f m}$ were negative for these three strains and one was ampicillin-resistant. Absence of IS16 among nonclinical E. faecium strains also suggests a very limited host range of IS16-bearing plasmids (e.g., pRUM-like) only among hospital-associated strains. Prevalence of hospitalassociated E. faecium isolates among the general human population is low and more detailed information was only available in one of the three cases. This outpatient from a community surveillance study received beta-lactam treatment during the time of sampling which could be the reason for identifying an ampicillin- and multiresistant, epidemic strain here (association with a hospital stay was not documented). 
An epidemiological analysis of 648 E. faecium clinical isolates sent to our Focal Laboratory for Enterococci in 2008/2009 from German hospital patients revealed that $98 \%$ ( $\mathrm{n}=637$ ) were IS16-positive, demonstrating countrywide prevalence of hospital-associated strain types among German hospitals in recent years. This finding was supported by wide prevalence of acquired high-level ciprofloxacin resistance among the same strain collection serving as an additional marker for hospital-associated strains [36]. Admittedly, most of the strains from 2008/2009 were vancomycin-resistant showing in retrospect that hospitalassociated strains of E. faecium and not commensal strain types accumulated in the nosocomial setting and acquired vanA- or vanB-type resistance determinants.

The observation that an IS element, IS16, is among the most specific markers to define hospital-associated strains [3] appears curious regarding the promiscuous nature of these elements to transpose between several genomic backgrounds within a cell and the possible horizontal mobilization in cis or trans by activation via conjugative elements and subsequent transfer into new hosts. It has been argued that acquisition of specific elements, especially IS16, has contributed to the ecological success of hospital-associated E. faecium strains allowing higher levels of genetic variability including easier acquisition of mobile genetic elements that may support lifestyle in new or changing environments or ecological niches like the hospital environment $[3,44]$.

\section{Conclusions}

Our data show that an IS16-based screening assay appears as the best molecular screening tool to differentiate colonizing E. faecium isolates from hospital-associated E. faecium strains that show an enhanced spreading potential in the nosocomial setting. The predictive value of an IS16 result is superior to the predictive strengths of established tests targeting molecular markers like esp or $h y l_{E f m}$ or phenotypic traits like acquired ampicillin or high-level ciprofloxacin resistance. Progress in comparative genome-based analysis will most probably reveal additional markers to identify hospital associated E. faecium isolates with an enhanced spreading potential in the nosocomial setting $[15,33]$.

\section{Additional material}

Additional file 1: Detailed information on the 160 clinical E. faecium isolates. The blood culture strains were part of this study and of Werner et al., [36]. "0" indicates absence, "1" indicates presence of the corresponding marker (esp, hyl Efm, IS16, vanA/B/O). Legend: AmpR, resistance to ampicillin; year, year of isolation.

Additional file 2: Detailed information on the 68 human commensal E. faecium isolates. The human commensal strains were part of several community prevalence studies performed in different parts of Germany and at different time periods (see text for details). "0" indicates absence,
"1" indicates presence of the corresponding marker (esp, hyl Efm, IS16, $\operatorname{van} A / B / 0)$. Legend: AmpR, resistance to ampicillin; year, year of isolation.

Additional file 3: Detailed information on the 32 animal $E$. faecium isolates. The animal strains were part of several prevalence studies performed in different parts of Germany and at different time periods (see text for details). " 0 " indicates absence, "1" indicates presence of the corresponding marker (esp, hyl Efm, IS16, vanA/B/0). Legend: AmpR, resistance to ampicillin; year, year of isolation.

Additional file 4: goeBURST analysis of all MLST types of the $E$. faecium database ( $\mathbf{n}=\mathbf{5 2 4}$ STs; 21.4.2010; http://efaecium.mlst.net/). Only the major, superior complex without singletons and smaller complexes is shown. A number of singletons from Figure 1 belong to clonal complexes such as ST19 which is part of the large cluster and related to CC17. Other complexes separated in Figure 1 (CC32, CC29) are linked here to form a main, superior cluster. ST65 remains a singleton and is the only IS16-positive ST not phylogenetically linked to the clonal complex of hospital-associated strains (CC17).

\section{Acknowledgements}

We kindly acknowledge all colleagues working in medical-microbiological laboratories and hospital laboratories sending their samples for further analyses to the Focal Laboratory for Enterococci, Germany, and apologize for being unable to list them all here. The study was partly supported by grants from EC-funded projects ("ACE", LSHE-CT-2007-037410; "TROCAR", LSHE-CT2008-223031)

Author details

${ }^{1}$ Robert Koch Institute, Wernigerode Branch, Wernigerode, Germany. ${ }^{2}$ University Medical Centre, Utrecht, The Netherlands.

\section{Authors' contributions}

IK manages and supervises the central enterococcal database and records all typing and characterization data from routine enterococcal diagnostics. UG performed standard PCRs, characterization and identification experiments. CF performed additional typing experiments such as PFGE, MLST and Southern hybridizations. WvS provided seven unpublished genome data and analyzed them regarding genetic linkages between IS16 and neighbouring regions. GW and WW supervised the study; GW selected the primer sequences, evaluated and calculated all the PCR, MLST, PFGE and hybridisation results. GW, IK and WW wrote the manuscript.

\section{Competing interests}

The authors declare that they have no competing interests.

Received: 6 May 2010 Accepted: 31 March 2011

Published: 31 March 2011

\section{References}

1. Arias CA, Murray BE: Antibiotic-resistant bugs in the 21st century - a clinical super-challenge. N Engl J Med 2009, 360:439-443.

2. Tenover FC: Development and spread of bacterial resistance to antimicrobial agents: an overview. Clin Infect Dis 2001, 33(Suppl 3): S108-S115.

3. Leavis HL, Willems RJ, van Wamel WJ, Schuren FH, Caspers MP, Bonten MJ: Insertion sequence-driven diversification creates a globally dispersed emerging multiresistant subspecies of E. faecium. PLoS Pathog 2007, 3:e7-75.

4. Willems RJ, van SW: Transition of Enterococcus faecium from commensal organism to nosocomial pathogen. Future Microbiol 2009, 4:1125-1135.

5. Lester CH, Sandvang D, Olsen SS, Schonheyder HC, Jarlov JO, Bangsborg J, Hansen DS, Jensen TG, Frimodt-Moller N, Hammerum AM: Emergence of ampicillin-resistant Enterococcus faecium in Danish hospitals. J Antimicrob Chemother 2008, 62:1203-1206.

6. Top J, Willems R, van d V, Asbroek M, Bonten M: Emergence of clonal complex 17 Enterococcus faecium in The Netherlands. J Clin Microbiol 2008, 46:214-219.

7. Nallapareddy SR, Singh KV, Okhuysen PC, Murray BE: A functional collagen adhesin gene, $a \mathrm{~cm}$, in clinical isolates of Enterococcus faecium correlates 
with the recent success of this emerging nosocomial pathogen. Infect Immun 2008, 76:4110-4119.

8. Sillanpaa J, Prakash VP, Nallapareddy SR, Murray BE: Distribution of genes encoding MSCRAMMs and pili in clinical and natural populations of Enterococcus faecium. J Clin Microbiol 2009, 47:896-901.

9. Hendrickx APA, Van Wamel WJB, Posthuma G, Bonten MJM, Willems RJL: Five genes encoding surface exposed LPXTG proteins are enriched in hospital-adapted Enterococcus faecium Clonal Complex-17 isolates. J Bacteriol 2007, 189:8321-8332

10. Hendrickx AP, Willems RJ, Bonten MJ, van SW: LPxTG surface proteins of enterococci. Trends Microbiol 2009, 17:423-430.

11. Freitas AR, Tedim AP, Novais C, Ruiz-Garbajosa P, Werner G, LaverdeGomez JA, Canton R, Peixe L, Baquero F, Coque TM: Global spread of colonization-virulence hyl $\mathrm{Efm}_{\mathrm{m}}$ gene in megaplasmids of $\mathrm{CC} 17$ Enterococcus faecium polyclonal sub-cluster. Antimicrob Agents Chemother 2010, 54:2660-2665.

12. Rice LB, Carias L, Rudin S, Vael C, Goossens H, Konstabel C, Klare I, Nallapareddy SR, Huang W, Murray BE: A potential virulence gene, hylefm, predominates in Enterococcus faecium of clinical origin. J Infect Dis 2003, 187:508-512.

13. Heikens E, Bonten MJM, Willems RJL: Enterococcal surface protein Esp is important for biofilm formation of Enterococcus faecium E1162. J Bacteriol 2007, 189:8233-8240

14. Willems RJ, Homan W, Top J, van Santen-Verheuvel M, Tribe D, Manzioros X, Gaillard C, Vandenbroucke-Grauls CM, Mascini EM, van KE, et al: Variant esp gene as a marker of a distinct genetic lineage of vancomycin-resistant Enterococcus faecium spreading in hospitals. Lancet 2001, 357:853-855

15. Heikens E, van Schaik W, Leavis HL, Bonten MJM, Willems RJL: Identification of a novel genomic island specific to hospital-acquired clonal complex 17 Enterococcus faecium isolates. Appl Environ Microbiol 2008, 74:7094-7097.

16. Lester CH, Olsen SS, Schonheyder HC, Hansen DS, Tvede M, Holm A, Arpi M, Friis-Moller A, Jensen KT, Kemp M, et al: Typing of vancomycinresistant enterococci obtained from patients at Danish hospitals and detection of a genomic island specific to $\mathrm{CC} 17$ Enterococcus faecium. Int $J$ Antimicrob Agents 2009, 35:312-314.

17. Nallapareddy SR, Weinstock GM, Murray BE: Clinical isolates of Enterococcus faecium exhibit strain-specific collagen binding mediated by Acm, a new member of the MSCRAMM family. Mol Microbiol 2003, 47:1733-1747.

18. Donabedian S, Thal LA, Bozigar P, Zervos T, Hershberger E, Zervos M: Antimicrobial resistance in swine and chickens fed virginiamycin for growth promotion. J Microbiol Methods 2003, 55:739-743.

19. McDonald LC, Rossiter S, Mackinson C, Wang YY, Johnson S, Sullivan M, Sokolow R, DeBess E, Gilbert L, Benson JA, et al: Quinupristin-dalfopristinresistant Enterococcus faecium on chicken and in human stool specimens. N Engl J Med 2001, 345:1155-1160.

20. Jung WK, Lim JY, Kwon NH, Kim JM, Hong SK, Koo HC, Kim SH, Park YH: Vancomycin-resistant enterococci from animal sources in Korea. Int $J$ Food Microbiol 2007, 113:102-107.

21. Klare I, Heier H, Claus H, Bohme G, Marin S, Seltmann G, Hakenbeck R, Antanassova $\mathrm{V}$, Witte W: Enterococcus faecium strains with vanA-mediated high-level glycopeptide resistance isolated from animal foodstuffs and fecal samples of humans in the community. Microb Drug Resist 1995, 1:265-272.

22. Klare I, Heier $H$, Claus $H$, Reissbrodt $R$, Witte W: vanA-mediated high-level glycopeptide resistance in Enterococcus faecium from animal husbandry. FEMS Microbiol Lett 1995, 125:165-171.

23. Klare I, Badstubner D, Konstabel C, Bohme G, Claus H, Witte W: Decreased incidence of VanA-type vancomycin-resistant enterococci isolated from poultry meat and from fecal samples of humans in the community after discontinuation of avoparcin usage in animal husbandry. Microb Drug Resist 1999, 5:45-52.

24. Lietzau S, Hoewner M, von BH, Marre R, Brenner H: Antibiotic resistant fecal isolates of Enterococci among unselected patients outside the clinical sector: an epidemiological study from Southern Germany. Pharmacoepidemiol Drug Saf 2006, 15:275-277.

25. Witte W, Werner G, Tietze E, Reissbrodt R, Klare I: Routes of transmission of antibiotic-resistant bacteria and their resistance determinants to humans via various environmental sources like crop food or bathing freshwater. Final report projekt UFOPLAN , 20162211, 2001 - 2004. 1-95. 1-3-2005.

26. Werner $G$, Klare I, Witte W: Large conjugative vanA plasmids in vancomycin-resistant Enterococcus faecium. J Clin Microbiol 1999 37:2383-2384
27. Klare I, Konstabel C, Mueller-Bertling S, Werner G, Strommenger B, Kettlitz C, Borgmann S, Schulte B, Jonas D, Serr A, et al: Spread of ampicillin/ vancomycin-resistant Enterococcus faecium of the epidemic-virulent clonal complex-17 carrying the genes esp and hyl in German hospitals. Eur J Clin Microbiol Infect Dis 2005, 24:815-825.

28. Freitas $A R$, Novais $C$, Ruiz-Garbajosa $P$, Coque $T M$, Peixe $L$ : Clonal expansion within clonal complex 2 and spread of vancomycin-resistant plasmids among different genetic lineages of Enterococcus faecalis from Portugal. J Antimicrob Chemother 2009, 63:1104-1111.

29. Feil EJ, Li BC, Aanensen DM, Hanage WP, Spratt BG: eBURST: inferring patterns of evolutionary descent among clusters of related bacterial genotypes from multilocus sequence typing data. J Bacteriol 2004, 186:1518-1530.

30. Francisco A, Bugalho M, Ramirez M, Carrico J: Global optimal eBURST analysis of multilocus typing data using a graphic matroid approach. BMC Bioinformatics 2009, 10:152.

31. Grady R, Hayes F: Axe-Txe, a broad-spectrum proteic toxin-antitoxin system specified by a multidrug-resistant, clinical isolate of Enterococcus faecium. Mol Microbiol 2003, 47:1419-1432

32. Dahl KH, Rokenes TP, Lundblad EW, Sundsfjord A: Nonconjugative transposition of the vanB-containing Tn5382-like element in Enterococcus faecium. Antimicrob Agents Chemother 2003, 47:786-789.

33. van Schaik W, Top J, Riley DR, Boekhorst J, Vrijenhoek JEPV, Schapendonk CME, Hendrickx APA, Nijman IJ, Bonten MJM, Tettelin H, et al: Pyrosequencing-based comparative genome analysis of the nosocomial pathogen Enterococcus faecium and identification of a large transferable pathogenicity island. BMC Genomics 2010, 11:239, BMC Genomics 2010.

34. Galloway-Pena JR, Nallapareddy SR, Arias CA, Eliopoulos GM, Murray BE: Analysis of clonality and antibiotic resistance among early clinical isolates of Enterococcus faecium in the United States. J Infect Dis 2009, 15:1566-1573

35. Leavis HL, Willems RJL, Top J, Bonten MJM: High-Level ciprofloxacin resistance from point mutations in $g y r A$ and parC confined to global hospital-adapted clonal lineage CC17 of Enterococcus faecium. J Clin Microbiol 2006, 44:1059-1064.

36. Werner G, Fleige C, Ewert B, Laverde-Gomez JA, Klare I, Witte W: High-level ciprofloxacin resistance among hospital-adapted Enterococcus faecium (CC17). Int J Antimicrob Agents 2010, 35:119-125.

37. Werner G, Coque TM, Hammerum AM, Hope R, Hryniewicz W, Johnson A, Klare I, Kristinsson KG, Leclercq R, Lester CH, et al: Emergence and spread of vancomycin resistance among enterococci in Europe. Euro Surveill 2008, 13.

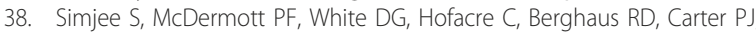
Stewart L, Liu T, Maier M, Maurer JJ: Antimicrobial susceptibility and distribution of antimicrobial-resistance genes among Enterococcus and coagulase-negative Staphylococcus isolates recovered from poultry litter. Avian Dis 2007, 51:884-892.

39. Top J, Willems R, Bonten M: Emergence of CC17 Enterococcus faecium: from commensal to hospital-adapted pathogen. FEMS Immunol Med Microbiol 2008, 52:297-308.

40. Willems RJ, Bonten MJ: Glycopeptide-resistant enterococci: deciphering virulence, resistance and epidemicity. Curr Opin Inf Dis 2007, 20:384-390.

41. Freitas $A R$, Novais C, Ruiz-Garbajosa P, Coque TM, Peixe L: Dispersion of multidrug-resistant Enterococcus faecium isolates belonging to major clonal complexes in different Portuguese settings. Appl Environ Microbiol 2009, 75:4904-4908.

42. Arias CA, Panesso D, Singh KV, Rice LB, Murray BE: Co-Transfer of antibiotic resistance genes and a hyl(Efm)-containing virulence plasmid in Enterococcus faecium. Antimicrob Agents Chemother 2009, 53:4240-4246.

43. Laverde-Gomez JA, van Schaik W, Freitas AR, Coque TM, Weaver $K_{\text {, }}$ Francia MV, Witte W, Werner G: A multiresistance megaplasmid bearing a hyl $\mathrm{l}_{\text {ffm }}$ genomic island in hospital Enterococcus faecium isolates. Int I Med Microbiol 2011, 301:165-175.

44. Burrus V, Waldor MK: Shaping bacterial genomes with integrative and conjugative elements. Research in Microbiology 2004, 155:376-386.

\section{Pre-publication history}

The pre-publication history for this paper can be accessed here: http://www.biomedcentral.com/1471-2334/11/80/prepub

doi:10.1186/1471-2334-11-80

Cite this article as: Werner et al:: IS element IS16 as a molecular screening tool to identify hospital-associated strains of Enterococcus faecium. BMC Infectious Diseases 2011 11:80. 